\title{
Abstracts From the NCCN 20th Annual Conference: Advancing the Standard of Cancer Care ${ }^{\mathrm{TM}}$
}

The following abstracts were accepted for presentation at the NCCN 20th Annual Conference: Advancing the Standard of Cancer Care $^{\mathrm{TM}}$ General Poster Session at The Westin Diplomat in Hollywood, Florida, on March 12-13, 2015.

\section{Outcomes and Health Services Research \\ AB2015-1. Anticipated Impact of Generic Imatinib Market Entry on Tyrosine Kinase Inhibitor-Related Pharmacy Costs Lisa M. Bloudek, PharmD, MSa; Dinara Makenbaeva, MD' ${ }^{\text {; }}$ and Michael Eaddy, PharmD, PhD ${ }^{a}$ \\ ${ }^{a}$ Xcenda and ${ }^{b}$ Bristol-Myers Squibb}

Background: GLEEVEC (imatinib; Novartis Pharmaceuticals Corporation, East Hanover, NJ) was the first tyrosine kinase inhibitor (TKI) approved for use in the United States and remains the most commonly prescribed BCRABL TKI, followed by SPRYCEL (dasatinib; Bristol-Myers Squibb, New York, NY) and TASIGNA (nilotinib; Novartis Pharmaceuticals Corporation, East Hanover, NJ). The upcoming patent expiration for GLEEVEC represents a significant cost savings potential for payers due to the availability of generic imatinib. The objective of this study was to estimate the economic impact of GLEEVEC loss of exclusivity (LOE) and formulary management with generic imatinib prior authorization (PA) before branded TKIs. Methods: An Excel-based model was developed from the US payer perspective over a period of 2 years. Use, patient out-of-pocket cost, and market share for TKIs constituting more than $5 \%$ of the TKI market were taken from claims data. Drug cost was based on wholesale acquisition cost, assuming 100\% adherence to standard dosing for chronic myelogenous lymphoma with no discontinuation. The cost of generic imatinib was assumed to be $47.8 \%$ of the GLEEVEC price. In the absence of formulary management, a shift from GLEEVEC to generic imatinib of $70 \%$ and $95 \%$ was assumed for years 1 and 2 after LOE, respectively, whereas market shares for SPRYCEL and TASIGNA held constant. For patients new to TKI therapy (assumed to be $16 \%$ for commercial and 34\% for Medicare), formulary management could be applied to require generic imatinib before a branded TKI. It was assumed that 74\% of PAs for branded TKIs were approved with an administrative cost of $\$ 20$ each. Outcomes included the change in total TKI-related pharmacy cost and per-member-permonth (PMPM) cost. Results: In a 1 million member commercial plan, GLEEVEC LOE alone was estimated to produce a cost savings of $\$ 6$ million during 2 years, or $28.6 \%$ of total pharmacy spending on the TKI class $(\$ 0.25$ PMPM). Greater savings were found in a Medicare plan, owing to higher TKI use rates ( $-\$ 20$ million or $-28.6 \%$; -\$0.85 PMPM). Formulary management reduced spending on TKIs by an incremental $1.0 \%$ and $2.2 \%$ for commercial and Medicare, respectively. Conclusions: In the absence of formulary management, GLEEVEC LOE is expected to reduce total pharmacy spending on TKIs by approximately one-third over 2 years. Given the low volume of patients treated with TKIs, formulary management of the TKI class has limited potential for incremental cost savings (1.0\% for commercial and 2.2\% for Medicare).

\section{Quality Improvement \\ AB2015-2. Improving Survivorship Care Provided by Primary Care Providers: Education Workshop Development in Response to Needs Assessment Survey Results Genevieve Chaput, MD, BA, MEd ${ }^{\text {a }}$, Desanka Kovacina, MD; Hitesh Bhanabhai, MD'; and Manuel Borod, $\mathrm{MD}^{\mathrm{a}}$ \\ aMcGill University Health Centre: Montreal General Hospital, and ${ }^{\text {bStillview }}$ Medical Clinic}

Background: Because of earlier detection and better treatments, the number of cancer survivors has increased significantly in the past 3 decades. Currently, there are approximately 1 million cancer survivors in Canada, and that number is expected to increase to 2 million by 2020 . In addition to higher risk of recurrence or second malignancy, $>50 \%$ of survivors are afflicted with late effects because of their cancer and its treatments. On completion of oncologic treatments, family physicians (FPs) are expected to play an integral role in the follow-up care of cancer survivors. However, literature findings show low confidence levels in FPs regarding survivorship care. In 2013-2014, our team conducted a survey targeting com- 
NCCN 20th Annual Conference Abstracts

munity FPs that was targeted to identify their needs and current perceived barriers in providing optimal care to cancer survivors. Our results showed the need for improved communication with specialists, and that education sessions should be provided on common issues of survivors. Methods: A brief survey containing Likert-type and short-answer questions was designed and distributed to FPs practicing within a McGillaffiliated center, family practice group, or private clinic in Montreal, Canada. The targeted sample size was 75 FPs. Survey questions were based on current practices, beliefs, knowledge, and needs. Data analysis included descriptive statistics and an open coding approach to identify major themes. McGill University Health Centre Research Ethics institutional approval was obtained. Results: Of 165 surveys distributed, 93 were completed, accounting for a response rate of $56.3 \%$. Of those completed, $94 \%$ of FPs were already caring for cancer survivors, and of these, $73 \%$ expressed a willingness to accept new patients with a history of cancer. A statistically significant number of FPs disagreed more than agreed that they were "receiving summary reports and follow-up plans" $(P=.001)$ and that they were "familiar with guidelines on surveillance" $(P=.01)$. Survey answers were not statistically influenced by "years of practice" and "practice setting" variables. The biggest barriers in providing care to cancer survivors were the lack of communication with specialists $(53 \%)$ and lack of knowledge of survivorship care (36\%). Conclusions: Various projects are ongoing at our institution to address the issues identified to optimize cancer survivor surveillance, including the development of a MAIN$\mathrm{PRO}$-accredited educational workshop targeting community FPs. This workshop, based on the 8 common issues of survivors as stated in the 2015 NCCN Guidelines for Survivorship, will be carried out at numerous FP sites, and include pretests and posttests to evaluate confidence and knowledge of survivorship care among the FPs. Preliminary data from 3 workshops will be available March 2015.

\section{Clinical Oncology \\ AB2015-3. A Phase I Study Afatinib/Carboplatin/ \\ Paclitaxel Induction Chemotherapy Followed by Standard Chemoradiation in HPV-Negative or High-Risk HPV- Positive Locally Advanced-Stage III/IVa/IVb HNSCC \\ Christine H. Chung, MDa; Hyunseok Kang, MDa; \\ Ana Kiess, MD, PhD; ; Harry Quon, MDa; Barbara Murphy, MD; and Jill Gilbert, MD \\ aJohns Hopkins University and ${ }^{b}$ Vanderbilt University \\ This study was approved and funded by the NCCN Oncology Research Program from general research support provided by Boehringer Ingelheim Pharmaceuticals, Inc.}

Background: Afatinib is an ErbB family receptor inhibitor that has shown efficacy in head and neck squa- mous cell carcinoma (HNSCC). A phase I trial was conducted to determine the maximum tolerated dose (MTD) or recommended phase II dose of afatinib in combination with carboplatin and paclitaxel as an induction regimen. Methods: Patients with human papillomavirus (HPV)-negative or those with significant smoking history and HPV-positive AJCC stage III, IVa, or IVb HNSCC of the oral cavity, oropharynx, hypopharynx, and larynx were enrolled. Oral afatinib was planned for a dose escalation from 20 to $40 \mathrm{mg}$ daily. Afatinib alone was given for 2 weeks as a lead-in and subsequently given with carboplatin at an area under the curve (AUC) of 6 and paclitaxel intravenously at $175 \mathrm{mg} / \mathrm{m}^{2}$ every 21 days as an induction regimen followed by concurrent intravenous cisplatin at $40 \mathrm{mg} / \mathrm{m}^{2}$ weekly and standard radiation. Toxicity was assessed using CTCAE version 4.0, and a standard $3+3$ design was used. Results: Nine patients ( 8 male, 1 female) were enrolled, with a mean age of 59.6 years, with primary sites of oral cavity $(n=4)$, oropharynx $(n=3)$, and larynx $(n=2)$. ECOG performance status was 0 for 6 patients and 1 for 3 patients. After completion of the induction regimen, 3 patients experienced a partial response and 4 had stable disease. Dose level 1 with afatinib at $20 \mathrm{mg}$ was well-tolerated, with one grade 3 (elevation in alanine transaminase levels) and one grade 4 (neutropenia) toxicities reported. However, dose level 2 with afatinib, $30 \mathrm{mg}$ was not well-tolerated, with nine grade 3 (pneumonia, abdominal pain, diarrhea, pancytopenia, and urinary tract infection), two grade 4 (sepsis), and one grade 5 (death) toxicities reported. Conclusions: The MTD of oral afatinib in combination with carboplatin and paclitaxel is 20 mg daily. Combination afatinib with carboplatin and paclitaxel should be administered with caution because of reported toxicities.

Outcomes and Health Services Research

AB2015-4. Real-World Treatment Patterns, Survival, and Costs in a US Medicare Population With Metastatic Squamous Non-Small Cell Lung Cancer

Keith L. Davis, MA; ; Ravi K. Goyal, MS, BPharm;

Stephen L. Able ${ }^{\text {b; Jacqueline Brown }}{ }^{\text {; }}$, Li Li ${ }^{\text {b; }}$ and

James A. Kaye, MD, DrPha

${ }^{a}$ RTI Health Solutions and ${ }^{b}$ Eli Lilly and Company

Background: Over the past decade, therapeutic choices for metastatic squamous non-small cell lung cancer (sqNSCLC) have not advanced to the same degree as those for non-sqNSCLC. Coupled with poor survival and continued growth of the elderly US population, assessment of current treatment patterns and care of patients with metastatic sqNSCLC is needed. Methods: A retrospective analysis of patients aged $\geq 65$ years with metastatic sqNSCLC diagnosed between 2001 
and 2009 was conducted using the SEER-Medicare database. Postdiagnosis data on treatment and costs of cancer-directed therapies, including chemotherapy, were descriptively analyzed. Multiple logistic regressions (MLRs) were conducted to identify predictors of treatment pattern events. Generalized linear models (GLMs) of all-cause and NSCLC-related costs were used to assess cost drivers. Median overall survival (OS) was estimated using Kaplan-Meier methods. Results: Of 17,133 patients eligible for study inclusion, $72 \%$ received some form of cancer-directed therapy; $45 \%$ overall received chemotherapy. Median OS was 8 months for patients receiving cancer-directed therapy versus 2 months in those receiving supportive care only $(P<.0001)$. MLR showed nearly 3-fold higher odds of receiving cancer-directed treatment in patients aged $65-74$ years versus those aged $\geq 85$ years $(P<.0001)$. Factors associated with decreased odds of receiving cancerdirected treatment were black versus white race (odds ratio [OR], 0.72; 95\% CI, 0.64-0.82), residence in the West versus South (OR, 0.73; 95\% CI, 0.66-0.81), metastatic disease at initial diagnosis versus progression to metastatic disease (OR, 0.77; 95\% CI, 0.70-0.84), and presence of several Charlson comorbidities. Mean total all-cause and NSCLC-related costs per patient for all available follow-up were $\$ 64,819$ (SD, $\$ 56,585)$ and $\$ 50,701$ (SD, $\$ 43,545)$, respectively. GLM regression on costs showed that patients aged 75-84 years receiving cancer-directed therapy had 10\% higher costs than those aged $\geq 85$ years. Other factors associated with significantly higher costs were black and other race (vs white) and Northeast and West geographic regions (vs South). A limitation of these analyses is that they are unadjusted for differences in baseline characteristics that may have contributed to differences in costs and outcomes. Conclusions: This study confirms that prognosis remains poor for patients with metastatic sqNSCLC. Direct per-patient medical costs of sqNSCLC are substantial, and with a growing elderly population in whom the peak incidence of this condition occurs, the cost burden on the Medicare system will likely continue to increase.

\section{Correlative/Genomic \\ AB2015-5. Testing for Inherited Cancer Pathogenic Variants in Women With Endometrial Cancer: Who to Test and For Which Genes to Test? \\ Dana Farengo-Clark, MS, CGC; Rachel Klein, MS, CGC; Megan Marshall, MS, CGC; Maegan Roberts, MS, CGC; Lisa Susswein, MS, MHA, CGC; Patricia Murphy, PhD, FACMG; and Kathleen Hruska, PhD, FACMG \\ GeneDx}

Background: Few studies address the familial risk of endometrial cancer (EC) outside of the mismatch repair
(MMR) genes (Lynch syndrome). PTEN and CHEK2 have also been reported in association with EC, but these risks are not well-defined. The role of other genes is currently unknown. Methods: We performed a retrospective review of clinical histories and genetic test results of women with a reported personal history of EC or uterine cancer tested at GeneDx from August 2013 through October $2014(\mathrm{~N}=489)$. Results: Of the 489 women with EC, $11.5 \%(n=56)$ were found to harbor a pathogenic or likely pathogenic $(\mathrm{P} / \mathrm{LP})$ variant. Not surprisingly, $60.7 \%(34 / 56)$ of the $\mathrm{P} / \mathrm{LP}$ variants were identified in an MMR gene: $28.6 \%(16 / 56)$ in MSH6, $14 \%(8 / 56)$ in MSH2, $10.7 \%(6 / 56)$ in MLH1, and $7.1 \%(4 / 56)$ in PMS2. P/LP variants in CHEK2 were found in $12.5 \%$ of women (7/56) with EC, of whom $85 \%(6 / 7)$ reported a family history of breast cancer. All of the women carrying a P/LP variant in either BRCA1 or BRCA2 $(12 \% ; 7 / 56)$ also reported a family history of breast cancer, and 1 of the 7 was diagnosed with breast cancer in addition to EC. The remaining women were found to harbor $\mathrm{P} / \mathrm{LP}$ variants in the following genes: ATM $(n=2)$, BRIP1 $(n=1)$, FANCC $(n=1)$, PALB2 $(n=1)$, PTEN $(n=1)$, RAD51C $(n=1)$, and MUTYH ( $\mathrm{n}=2$, compound heterozygotes). Of all women with an MMR P/LP variant, 38\% (13/34) had a separate primary Lynch syndrome cancer in addition to EC. Furthermore, $61 \%$ of women (21/34) with an MMR P/LP variant reported a family history of colon cancer, and 32.4\% (11/34) met Amsterdam II criteria. Stratifying by age, women diagnosed before and after age 50 years were found to carry a pathogenic variant with similar frequency $(11.3 \%$ [13/115] vs $11.5 \%$ [43/374], respectively). However, pathogenic MMR gene variants accounted for most variants $(92.0 \%$ [12/13]) identified in the younger cohort, whereas a lower and statistically significant $(P=.0091)$ proportion of $M M R$ pathogenic variants were identified in women diagnosed at $>50$ years of age (51\% [22/43]). Conclusions: Current NCCN Guidelines for endometrial cancer (in the NCCN Guidelines for Uterine Neoplasms, available at NCCN.org) are focused on Lynch syndrome and recommend that genetic counseling/testing be considered in those diagnosed at $<50$ years of age. We found that $39.3 \%$ of identified pathogenic variants were in genes not traditionally associated with EC, and that the presence of a pathogenic variant was similar in women both older and younger than 50 years of age. Genetic testing via a multigene cancer panel could be considered for all women with a personal history of $\mathrm{EC}$ who report a family history of colon and/or breast cancer, regardless of their age at diagnosis, to increase the likelihood of identifying an underlying hereditary predisposition. 
NCCN 20th Annual Conference Abstracts

\section{Correlative/Genomic}

AB2015-6. Genetic Approaches for Dissecting the Role of MLL2 in Tumorigenesis

Yiping $\mathrm{He}$, PhD

Duke Cancer Institute, The Preston Robert Tisch Brain Tumor Center and Pediatric Brain Tumor Foundation Institute, Department of Pathology, Duke University

Background: Recent studies in cancer genetics have revealed that chromatin remodeling and histone methylation modifiers are frequently altered in human cancers. Among these newly identified cancer genes, one of the best examples is the gene encoding MLL2 (KMT2D; ie, ALR/MLL4), a histone lysine methyltransferase that plays an important role in regulating gene transcription. Genetic alterations suggestive of a functional deficiency in MLL2 and other genes in the same pathways are common. The identification of alterations in MLL2 suggests potential new opportunities for therapeutics, and highlights an urgent need to understand the underlying tumorigenic mechanism. Filling such a knowledge gap has been challenging because of the lack of appropriate assays for the gigantic $(\approx 600$ $\mathrm{kDa}$ ) and understudied MLL2 protein. Methods: Somatic gene editing-based and gene knockout-based assays were used to determine the effect of an MLL2 deficiency on neoplastic cells. Particularly, homologous recombination- and nuclease-mediated gene editing approaches were used to generate a panel of isogenic human colorectal cancer and medulloblastoma cell lines that differ with respect to their endogenous MLL2 status. In addition, primary cell models with MLL2 deficiency were generated to study the role of MLL2 in initial cell transformation. Results: These studies found that an MLL2 deficiency results in attenuated cancer cell proliferation and defective cell migration. Direct transcriptional target genes were identified, which revealed the connection of MLL2 to multiple cellular signaling pathways, including the $\mathrm{p} 53$ pathway. Analysis of histone $\mathrm{H} 3$ modifications revealed that MLL2 is essential for maintaining the level of global histone $\mathrm{H} 3$ lysine 4 (H3K4) monomethylation, and that its enzymatic SET domain is directly responsible for this function. Findings showed that most MLL2 binding sites are located in regions of potential enhancer elements. The finding about enhancer elements is a significant advance, because enhancer elements have increasingly been recognized as being critically involved in tumorigenesis. Conclusions: Together, these findings revealed the role of MLL2 in mediating diverse signaling pathways and regulating enhancer elements in human cells, and shed light on the tumorigenic role of its deficiency. This study supports that MLL2 has distinct roles in different stages of cancer development, and that inhibiting MLL2 may be a viable strategy for can- cer therapeutics. These studies also established a gene editing-based approach for studying other chromatin remodeling and histone methylation modifiers that play a role in tumorigenesis.

Best Practices in Implementation and Use of

Clinical Practice Guidelines

AB2015-7. Evidence-Based Decision-Making to Reduce

Variation in the Treatment of Stage I Lung Cancer in a Community-Based Health System

Lee Kamman, MD; Anna Briggs, PhD, MPH

Matthew Graczyk, MDa,b; Connie Fiebiger, RN, MA;

Andrew Crow, MLISa; Jill Trei, MPH'; Jacqueline Yates, MPH'; and Timothy Sielaff, MD

${ }^{\mathrm{a}}$ Allina Health and ${ }^{\mathrm{b}} \mathrm{MN}$ Oncology

Background: The Lung Program Committee (LPC) is a multidisciplinary group of experts within the Virginia Piper Cancer Institute Clinical Service Line that drives best clinical practice through system integration within Allina Health (Allina). Variation exists in the treatment (surgery vs radiation) of stage I non-small cell lung cancer (NSCLC) because of the subjective determination of high-risk status (ie, operability). Currently, no clinical pathway for determining high-risk status for surgery among patients with stage I NSCLC has been implemented throughout Allina. To address this variation in practice, we aimed to (1) define objective criteria for determining "high risk" and implement a clinical pathway for stage I NSCLC, and (2) demonstrate the value of the Allina model for Evidence-Based Decision-Making (EBDM) within the LPC. Methods: Data on patients diagnosed with stage I NSCLC from 2012 through 2013 in 3 metropolitan area hospitals were obtained from the Allina Health Tumor Registry. Patients who received surgery, radiation, or no treatment were compared with expected values using chi-square analysis for all patients and potentially high-risk patients. The Allina EBDM model was used to develop a clinical pathway. Results summarize variation in treatment by site and the utility of the Allina Health EBDM process in reducing variation related to treatment of stage I NSCLC. Timeline for implementation is also presented. Results: Table 1 shows statistically significant differences in treatment type at the 3 separate sites among all patients diagnosed with stage I NSCLC (N=349; Analysis 1) and among potentially high-risk patients $(\mathrm{N}=166$; Analysis 2). Performance improvement outcomes from the EBDM process include the development of 1 multidisciplinary expert panel, 2 systematic reviews, 1 performance improvement charter, and 1 best-evidence clinical pathway for stage I NSCLC. The pathway includes an LPC endorsement of best practice recommendations published by the American College of Chest Physicians 
(Howington JA, et al; Chest 2013) and NCCN (Ettinger DS, et al; NCCN Guidelines for Non-Small Cell Lung Cancer; NCCN.org). The performance charter committed resources within Allina to define, standardize, and implement criteria for determining high-risk status, and includes an outcome metric of $\leq 10 \%$ difference in treatment of patients with stage I NSCLC and process metrics regarding referral patterns, complications, and recurrence rates. The implementation timeline includes data abstraction by December 2014 and 3 months of postimplementation data by March 2015. Conclusions: The Allina EBDM process shows high value and feasibility within the LPC for the treatment of stage I NSCLC, indicating the potential for process improvements and to provide consistent care across a system of independent, multispecialty physicians.

\section{AB2015-7. Table 1: Variation in Treatment of NSCLC by} Center* (2012-2013)

\begin{tabular}{|c|c|c|c|}
\hline \multirow[t]{2}{*}{ Analysis 1} & \multicolumn{3}{|c|}{$\begin{array}{l}\text { All Patients With Stage I NSCLC } \\
(\mathrm{N}=349)\end{array}$} \\
\hline & $\begin{array}{l}\text { Site } A \\
(N=137)\end{array}$ & $\begin{array}{l}\text { Site } B \\
(N=108)\end{array}$ & $\begin{array}{l}\text { Site C } \\
(N=104)\end{array}$ \\
\hline $\begin{array}{l}\text { Surgery, all types } \\
(\%)\end{array}$ & $87(63.5)^{\dagger}$ & $80(74.1)$ & $95(91.3)^{\ddagger}$ \\
\hline $\mathrm{RT}$, all types (\%) & $39(28.5)^{\ddagger}$ & $19(17.6)$ & $4(3.8)$ \\
\hline No treatment $(\%)$ & $11(8.0)$ & $11(10.2)$ & $5(4.8)$ \\
\hline Analysis 2 & \multicolumn{3}{|c|}{$\begin{array}{l}\text { Potentially High Risk Patients }{ }^{\S} \\
(n=166)\end{array}$} \\
\hline $\begin{array}{l}\text { Surgery, sublobar } \\
(\%)\end{array}$ & $24(32.4)^{\dagger}$ & $13(31.9)^{\dagger}$ & $36(80)^{\ddagger}$ \\
\hline RT, stereotactic (\%) & $30(46.2)$ & $15(39.5)^{\ddagger}$ & $1(2.4)^{\dagger}$ \\
\hline No treatment (\%) & $11(14.9)$ & $11(23.4)$ & $5(11.1)$ \\
\hline
\end{tabular}

Abbreviation: RT, radiation therapy.

${ }^{*}$ Sites B and C are independently owned radiation centers, therefore some cases may not have been captured.

'Less than expected, $P<.001$.

${ }^{\ddagger}$ More than expected, $P<.001$.

${ }^{\S}$ Of 349 patients diagnosed with stage I NSCLC, 166 (47.6\%) received either sublobar resection, stereotactic radiation, or no treatment indicating potentially high risk.

\section{Epidemiology/Risks}

\section{AB2015-8. Use of Real-World Claim Databases to Assess} Comorbidities Relevant to the Treatment of CML Based on NCCN Guidelines

Elias Jabbour, MD; Dinara Makenbaeva, MD, MBA;

Melissa Lingohr-Smith, $\mathrm{PhD}^{\mathrm{c}}$; and Jay Lin, $\mathrm{PhD}, \mathrm{MBA}^{\mathrm{c}}$

${ }^{a}$ The University of Texas MD Anderson Cancer Center, ${ }^{b}$ Bristol-Myers Squibb, and 'Novosys Health

Background: Three BCR-ABL tyrosine kinase inhibitors (TKIs), imatinib, dasatinib, and nilotinib, have a category 1 recommendation from the NCCN Guidelines for Chronic Myelogenous Leukemia (CML) for the treatment of this patient population. The NCCN Guidelines for CML state that, based on toxicity profiles, nilotinib may be preferred for patients with CML who have a history of lung disease or are at risk for pleural effusions, whereas dasatinib may be more appropriate for those with a history of arrhythmias, heart disease, pancreatitis, or hyperglycemia. The objective of this study was to assess the prevalence of comorbidities relevant to TKI treatment among patients with CML in US real-world settings. Methods: Patients with CML who had a newly initiated TKI treatment were identified from the Truven Health Analytics Commercial and Medicare MarketScan databases between January 1, 2006, to June 30, 2013. These databases contain data for $>66$ million covered individuals, and allow for the study of a nationally representative sample of Americans with employer-provided health insurance. First TKI prescription was defined as the index event. Patients were required to have $\geq 12$ months of continuous data before the index event, defined as the baseline period. Demographics and the prevalence of comorbidities relevant to TKI treatment choice (heart disease, arrhythmia, diabetes, pancreatitis, pleural effusion, and lung disease) were assessed. Results: Median age of the CML study population newly initiated on TKI treatment $(\mathrm{N}=2296)$ was 56 years and $77 \%(1766)$ were $<65$ years of age. Among the study population $38 \%$ were from the South, 30\% were from the North Central region, 16\% were from the West, and 14\% were from the Northeast. Approximately $41 \%$ of the CML study population had $\geq 1$ comorbidity that may influence the choice of TKI treatment as recommended in the NCCN Guidelines (Table 1 ). The most prevalent comorbidity was heart disease (22.8\%), followed by diabetes (17.8\%) and lung disease (12.7\%). Conclusions: This large-scale retrospective claims analysis assessed the real-world prevalence of the comorbidities mentioned in the NCCN Guidelines as having relevance to TKI treatment choice. We observed that the prevalence of these comorbidities is considerable among patient with CML and should be taken into account when choosing the most appropriate TKI treatment.

\section{AB2015-8. Table 1: Prevalence of Comorbidities Among the CML Study Population}

\begin{tabular}{|lrr|}
\hline Condition & N & \multicolumn{1}{c|}{$\%$} \\
\hline Heart disease & 523 & 22.8 \\
Arrhythmia & 152 & 6.6 \\
Diabetes & 409 & 17.8 \\
Pancreatitis & 8 & 0.4 \\
Pleural effusion & 47 & 2.1 \\
Lung disease & 292 & 12.7 \\
Any of the above & 937 & 40.8 \\
\hline
\end{tabular}


NCCN 20th Annual Conference Abstracts

Best Practices in Implementation and Use of

Clinical Practice Guidelines

\section{AB2015-9. Longitudinal Evaluation of a Colorectal Cancer} Screening Measurement Model

Bryan Loy, MD MBA; William T. Brooks, BS; Michael Yang, MD, MS; Laura E. Happe, PharmD, MPH; and

Thomas J. Van Gilder, MD, JD, MPH

Humana, Inc.

Background: The American College of Gastroenterology recommends a colonoscopy be performed every 10 years in individuals aged $\geq 50$ years for the prevention of colorectal cancer (CRC). Evidence-based alternatives include flexible sigmoidoscopy (FSG) and fecal occult blood tests (FOBT). Although CRC is the leading cause of cancer deaths in the United States, the CDC reports that $35 \%$ of adults aged 50 to 75 years are not current with screenings. Accordingly, a national health care company implemented a multifaceted strategy targeted at improving CRC screening rates. Each day, an analytic engine assesses data from the entire population against eligibility criteria for CRC screenings. Flags are generated for eligible individuals, and a variety of screening advocacy outreach strategies are used. Methods: Given the opportunity for outreach over time, this observational study evaluated the longitudinal relationship between CRC screening compliance and the length of time an individual was eligible for screening advocacy outreach. Using administrative claims data from a health care company insuring $>2.8$ million Medicare Advantage members (2nd quarter 2014), the analytic engine identified people aged 65 to 76 years who were eligible for CRC screening on a minimum of 1 day between June 1, 2013, and May 31, 2014. Compliance with CRC screenings was defined by the presence of a claim for colonoscopy, FSG, or FOBT within the recommended time frame. Compliance was reported for the eligible population, stratified by sex and the length of time an individual was eligible for screening advocacy outreach (ie, insured by the plan and eligible for screening). Chi-square tests for significance were used. Results: The sample population $(\mathrm{N}=1,017,024)$ was $55.0 \%$ female with a mean age of 69.6 years. Overall, $56.0 \%$ of the population was compliant with screenings, with colonoscopy being the most common test $(82.6 \%)$. Women were more compliant with screenings than men $(58.2 \%$ vs $54.5 \% ; P<.001)$. CRC screening rates stratified by the length of time eligible for screening advocacy outreach are shown in Table 1 ( $P<.001$ for all time points). Conclusions: Compliance with CRC screening guidelines was higher among those who were eligible for screening advocacy outreach for a longer period. Additional research is needed to determine whether the implementation of outreach strategies over time influenced this observed trend. These findings support the concept that longitudinal relationships with patients are preferred over the fragmented models.

\section{AB2015-9. Table 1: CRC Screening Rates Stratified by Screening Advocacy Outreach}

\begin{tabular}{|ll|}
\hline Length of Time & $\begin{array}{l}\text { CRC Screening } \\
\text { Compliance Rates }\end{array}$ \\
\hline Any length of time $(\mathrm{N}=1,017,024)$ & $56.0 \%$ \\
1 y $(1-365 \mathrm{~d})$ & $47.6 \%$ \\
2 y $(366-730 \mathrm{~d})$ & $51.7 \%$ \\
3 y $(731-1095 \mathrm{~d})$ & $61.8 \%$ \\
$>3$ y $(1096-1381 \mathrm{~d})$ & $65.5 \%$ \\
\hline
\end{tabular}

Clinical Oncology

AB2015-10. Five-Year Analysis of a Multiinstitutional Prospective Clinical Trial of Delayed Intervention and Surveillance for Small Renal Masses: The DISSRM Registry

Phillip M. Pierorazio, MDa; Bruce J. Trock, PhDa; Peter Chang, $\mathrm{MD}^{\mathrm{b}}$; Andrew Wagner, $\mathrm{MD}^{\mathrm{b}}$; James M. McKiernan, $\mathrm{MD}^{\mathrm{c}}$; and Mohamad E. Allaf, MD ${ }^{\mathrm{a}}$

aThe James Buchanan Brady Urological Institute and Department of Urology, Johns Hopkins Medicine; ' ${ }^{b}$ Division of Urology, Beth Israel Deaconess Medical Center; and 'Department of Urology, Columbia University Medical Center

Background: A growing body of retrospective literature has emerged regarding active surveillance (AS) for patients with small renal masses (SRM). There are limited prospective data evaluating the effectiveness of AS compared with primary intervention (PI). Therefore, we sought to determine the patient characteristics and clinical outcomes of patients who elected to undergo AS for management of their SRM. Methods: From 2009 to 2014, the multiinstitutional Delayed Intervention and Surveillance for Small Renal Masses (DISSRM) registry prospectively enrolled 496 patients with renal masses $\leq 4.0 \mathrm{~cm}$ who chose PI or AS. The registry was designed and powered as a noninferiority study. Objective criteria gleaned from existing literature (DISSRM score) were applied to all AS participants. Analyses were performed in an intention-to-treat manner. Primary outcomes were overall and cancer-specific survival. Results: The number of patients electing AS, PI, and crossover to delayed intervention was 274 (55.2\%), 222 (44.8\%), and $21(4.1 \%)$, respectively. AS patients were older, had worse ECOG scores, total comorbidities, cardiovascular comorbidities, had smaller tumors, and more often had multiple and bilateral lesions. Average DISSRM score was 1.93 and 3.42 in the PI and AS cohorts, respectively $(P=.04)$. At 4 years (median follow- 
up, 2 years), overall survival was $92.0 \%$ and $89.9 \%$ $(P=.06)$, and cancer-specific survival was $98.5 \%$ and $100 \%$, respectively $(P=.34)$._Conclusions: In a wellselected cohort with up to 5 -years of prospective follow-up, AS is not inferior to PI. The DISSRM Score is a useful objective scoring method to differentiate patients with SRM most suitable for AS.

Best Practices in Implementation and Use of

Clinical Practice Guidelines

\section{AB2015-11. Oncotype DX Use in Breast Cancer Cases} Reported in SEER (2010-2012)

Valentina Petkov, MD, MPH; Nadia Howlader, MS; Lynne Penberthy, MD, MPH; and Kathy Cronin, PhD, MPH NIHINCIIDCCPS/Surveillance Research Program

Background: Since 2008, NCCN Guidelines for Breast Cancer (BC) have recommended that Oncotype DX testing be considered in early-stage BC (pathologic tumor stage pT1, pT2, or pT3; lymph node-negative or lymph node-positive for micrometastases (N1mi); hormone receptor-positive; HER2 $^{-}$; and tumor size $>0.5 \mathrm{~cm}$ ). The recurrence score (RS) can help identify patients at high risk for recurrence and who may benefit from chemotherapy. Multigene assays testing (including Oncotype DX) have been required for collection by SEER cancer registries since 2010. This study assessed Oncotype DX use according to guidelines. Methods: SEER data for 2010-2012 was analyzed to evaluate Oncotype DX use, RS distribution, and chemotherapy use. Logistic regression modeling was used to determine whether demographic and tumor characteristics were associated with performing the test in patients who met guidelines criteria. Results: There were 225,065 incident cases of BC in the SEER 2010-2012 data and, of those, 25,411 (11.3\%) underwent Oncotype DX testing. An additional $0.7 \%$ had MammaPrint or other tests. Of the 71,742 patients who met the guideline criteria for testing, 18,940 (26.7\%) underwent Oncotype DX testing. A slight increase in testing was seen from 2010 to 2012 (23.3\% vs 29.1\%, respectively), and $75 \%$ of all Oncotype DX tests were performed according to guidelines. Increased use of the test was found in patients with node-positive BC and ductal carcinoma in situ, from 22\% of all tests in 2010 to $32 \%$ in 2012. Patient distribution according to RS risk category was $54.6 \%$ low risk (RS, $0-17$ ), $34.3 \%$ intermediate risk (RS, 18-30), and 9.1\% high risk (RS, 31-100). Chemotherapy was given to $5.4 \%$ of patients in the low-risk, $36.4 \%$ in intermediate-risk, and $77.4 \%$ in the high-risk categories, and in $17.0 \%$ of patients who did not undergo Oncotype DX testing. No racial or insurance status differences were seen between those who were tested versus those who were not. Women that were tested were more likely to be younger (odds ratio [OR], 7.54 [7.02-8.11] for age $50-64$ vs $\geq 75$ years), married (OR, 1.35 [1.271.49] for married vs widowed;), and had moderately differentiated tumors. Conclusions: Oncotype DX testing in SEER data seems to follow guidelines with risk stratification predicting use of chemotherapy in early-stage BC.

\section{Outcomes and Health Services Research \\ AB2015-12. Comparing Costs and Health Outcomes in Initial Therapy With Sunitinib Versus Pazopanib for Renal Cell Carcinoma}

Patrick Racsa, MSa; Karen Worley, PhDa; and

Tyler Whisman, PharmD

${ }^{a}$ Comprehensive Health Insights, Humana Inc., and ${ }^{b}$ Humana Inc

Background: Sunitinib and pazopanib are firstline treatments for advanced renal cell carcinoma (RCC), yet real-world, head-to-head comparisons of treatment effectiveness are lacking. This study compared adherence, mortality, and overall health care costs in sunitinib versus pazopanib. Methods: Administrative claims data from a national health plan was used to retrospectively identify individuals with medical and pharmacy insurance (commercial or Medicare); were aged 18-89 years; had $\geq 1$ pharmacy claim for sunitinib or pazopanib between November 1, 2009, and December 31, 2012; were diagnosed with RCC (ICD-9-CM 189.0 or 189.1); and had $\geq 6$ months of continuous enrollment before the initial pharmacy claim (index date). Patients receiving either sunitinib or pazopanib in the 6 months before the index date or without 12 months continuous coverage after the index date (unless terminated by death) were excluded. Primary outcomes were treatment adherence (calculated as proportion of days covered [PDC]), mortality, and costs. Statistical tests used for unadjusted and adjusted models included: $t$ tests, chi-square tests, and regression models. Results: Among the 353 patients included in the study, there were no statistically significant differences by index therapy in age, gender, race, region, or insurance plan type. Patients on pazopanib had worse baseline health status indicators based on a higher RxRisk$\mathrm{V}$ score $(P=.015)$ and had $\$ 8,000$ higher average costs $(P=.020)$. Postindex adherence was low and similar in both groups, with fewer than one-third of patients achieving PDC $\geq 80 \%$ (Table 1). Nearly half of the patients died during follow-up, with no differences by treatment group. Overall, postindex health care costs trended higher for patients 
on sunitinib $(P=.069)$. Among nonadherent patients, the difference was significantly higher among those on sunitinib ( $\$ 82,720$ vs $\$ 65,040$ for pazopanib; $P=.012$ ), although both medical and overall costs for adherent patients were similar between the drugs. Conclusions: Adherence and mortality were comparable between medications, but postindex costs were higher with sunitinib despite indicators of poorer health at baseline for patients on pazopanib. Nonadherence was common in both groups and was associated with significantly higher costs in the sunitinib group. More research is needed to validate this work and to better understand potential drivers in the observed cost differences.

\section{AB2015-12. Table 1: Summary of Results}

\begin{tabular}{|lclll|}
\hline Outcome & Sunitinib & Pazopanib & RR & P Value* \\
\hline $\begin{array}{l}\text { Adherence } \\
\text { PDC, mean }\end{array}$ & $0.52( \pm 0.31)$ & $0.44( \pm 0.32)$ & - & .201 \\
$\begin{array}{l}\text { (SD) } \\
\text { PDC } \geq 80 \%,\end{array}$ & $68(28.2 \%)$ & $23(20.5 \%)$ & 1.37 & .169 \\
$\mathrm{n}(\%)$ & & & \\
$\begin{array}{l}\text { Mortality, n } \\
(\%)\end{array}$ & $87(45.1 \%)$ & $41(45.6 \%)$ & 1.05 & .706 \\
$\begin{array}{l}\text { Postindex costs, mean } \\
\text { Overall }\end{array}$ & & & \\
Adherent & $\$ 76,624$ & $\$ 64,432$ & - & .069 \\
Nonadherent & $\$ 82,720$ & $\$ 65,040$ & - & .012 \\
\hline
\end{tabular}

Abbreviation: $\mathrm{RR}$, relative risk.

*Adjusted for covariates.

Best Practices in Implementation and Use of Clinical Practice Guidelines

AB2015-13. Establishing Psychosocial Standards of Care for Childhood Cancer

Victoria Sardi-Brown, PhD, LPC; ;eter J. Brown, MBA; Lori Wiener, PhD, DCSW'; Mary Jo Kupst, $\mathrm{PhD}^{\text {c; }}$;

Anne Kazak, PhD, ABPPd; Robert B. Noll, PhDe; and Andrea Patenaude, $\mathrm{PhD}^{f}$

${ }^{a}$ Mattie Miracle Cancer Foundation, ${ }^{b} \mathrm{NCl}, \mathrm{NIH}$, ${ }^{c}$ Medical College of Wisconsin, ${ }^{d}$ Nemours Children's Health System and Sidney Kimmel Medical School of Thomas Jefferson University, eUniversity of Pittsburgh, and ${ }^{f}$ Dana-Farber Cancer Institute

Background: Psychosocial care is an essential component of comprehensive care for people diagnosed with cancer (IOM, 2008). With the demand for accountability and outcome-driven, cost-effective models, psychosocial clinicians are being challenged to standardize their approach and evaluate the efficacy of their clinical efforts (Noll et al., 2013; Wiener et al., 2014). In 2013, the Psychosocial Standards of Care Project for Childhood Care (PSCPCC) was initiated to develop evidence-based clinical guidelines for the psychosocial care of children with cancer and their families from diagnosis through survivorship or end-of-life and bereavement care. Methods: Extensive literature reviews of existing standards, guidelines, and consensus reports were conducted in conjunction with an online survey exploring the perceived needs of children with cancer and their families in all settings in which a child with cancer could be treated. These data informed work conducted at a think tank (2013) with national and international pediatric psychosocial oncology experts, and a consensus was determined regarding what the "essential" elements (Reckis et al., 2011) should be for pediatric psychosocial care. After the think tank, 4 working groups consisting of 22 psychologists, 3 psychiatrists, 5 social workers, 1 nurse, 2 oncologists, and 5 parents from the United States, Canada, and The Netherlands had regular conference calls to review the literature and ensure evidence supported each of the 26 elements generated. The Appraisal Guidelines for Research and Evaluation (AGREE-II, 2003) was followed. Each element was sent out for external review to pediatric oncologists and psychosocial clinicians. Results: At a second think tank (2014), a comprehensive document of the 26 essential elements, evidence summaries, and rating forms were reviewed by pediatric psychosocial oncology experts. A consensus-based approach was used, and recommended standards without sufficient evidence were eliminated. The revised list comprises 19 standards. Next steps involve continued review of evidence for quality and rigor using the GRADE approach (Guyatt, 2008), further vetting from professional and advocacy organizations and stakeholders, and the development of algorithms to improve guideline dissemination and use. Conclusions: PSCPCC is committed to creating and disseminating a 21st century, widely applicable blueprint to support and advocate for universally available psychosocial services (Wiener et al., 2014).

\section{Preclinical Oncology}

AB2015-14. Detection of Stage I-IV Ovarian Cancer From a Drop of Serum

Theodore M. Tarasow, PhD; Mojgan Haddad, PhD; Joseph Barton Legutki, PhD; Scott A. Melville, PhD; Owen Solberg, PhD; and Kathryn Sykes, PhD HealthTell, Inc.

Background: Ovarian cancer remains the most lethal gynecologic disease and the second most common cause of female cancer deaths in Western countries, largely because it is found and diagnosed at later stages. No recognized and reliable diagnostic tools are currently available to effectively screen for ovarian cancer, even in high-risk populations in which the probability of a posi- 
NCCN 20th Annual Conference Abstracts

tive diagnosis would be highest. Our laboratories have developed a new concept for disease detection based on immunosignatures. Immunosignature technology uses arrays of hundreds of thousands of unique peptides designed to broadly survey an patient's antibody binding repertoire from a drop of blood, serum, or plasma. Arrayed library peptides that bind differentially between case and control samples are selected as input into classification algorithms that are then trained against cohorts of samples representing intended-use populations. Immunosignature technology holds promise for detecting the presence of virtually any disease that generates a significant B-cell response. For cancer, immunosignature technology has been shown to be capable of distinguishing patient samples representing several different cancers from each other and from noncancer controls, with high accuracy. Methods: To determine the feasibility of using immunosignature technology for detecting ovarian cancer, a cohort of samples representing 39 pathology-confirmed cases of ovarian cancer (stage I, III, and IV cancers, pretreatment) and 29 age- and smoking history/status-matched controls were assayed on high-density peptide arrays. Peptides with a statistically significant difference in binding signal between the cases and controls were identified by $t$ test with Bonferroni adjusted $P$ value. A support vector machine model was trained as the classification algorithm using the 100 most significant features. Classification performance characteristics were evaluated by applying 100 iterations of a 4-fold cross-validation routine. The cross-validated performance metrics of the 100-feature immunosignature classifier were determined. Results: The area under the receiver operator curve was 0.95 , sensitivity was $88 \%$, specificity was $91 \%$, and overall accuracy was determined to be $90 \%$. Notably, the samples from patients with stage I ovarian cancer were more accurately classified than samples from patients with later-stage disease. Conclusions: These results need verification in larger cohorts and validation in blinded studies; however, they are deemed promising for the early detection of ovarian cancer. The accuracy in classifying early-stage disease samples indicates that immunosignatures may be well-suited to enable detection and therefore treatment of early-stage ovarian cancer.

\section{Outcomes and Health Services Research \\ AB2015-15. Current Treatment Characteristics in Postmenopausal Women With HR+/HER2- Metastatic Breast Cancer}

Francis Vekeman, MAa; Yanni Hao, $\mathrm{PhD}^{\mathrm{b}}$; Wendy Cheng, MPH, MPhil; Jonathan Fortier, MA'; Marie-Noëlle Robitaille, MA'; and Mei Sheng Duh, MPH, SCD

aGroupe d'analyse, Ltée., 'Novartis Pharmaceuticals Corporation, and 'Analysis Group, Inc.
Background: With BOLERO-2 clinical trial data suggesting greater efficacy with everolimus in combination with exemestane in postmenopausal women with hormone receptor-positive $\left(\mathrm{HR}^{+}\right)$/human epidermal growth factor receptor-positive (HER2-) metastatic breast cancer ( $\mathrm{mBC})$, guidance on use and selection of endocrine therapy (ET) is of increasing clinical importance. This study aimed to present an up-to-date characterization of treatment patterns in patients with $\mathrm{HR}^{+} / \mathrm{HER} 2^{-} \mathrm{mBC}$, with an emphasis on patients who received first-line ET versus first-line chemotherapy in real-world settings. Methods: Data were extracted from a community oncology electronic medical records database from Altos Solutions, Inc. Eligible patients were postmenopausal, had $\geq 1$ medical record with a breast cancer diagnosis (ICD-9,174.xx), and had confirmed $\mathrm{HR}^{+} \mathrm{HER}^{-} \mathrm{mBC}$. The index date was defined as the date of first $\mathrm{mBC}$ diagnosis. Treatment patterns were compared for patients with first-line ET versus first-line chemotherapy and across therapy lines. Results: A total of 1218 patients met the inclusion criteria (first-line ET, 843 [69\%]; first-line chemotherapy, 375 [31\%]). Compared with first-line chemotherapy, patients who received first-line ET were older (mean age, 69 vs 64 years; $P<.001)$ and were more likely to have Medicare coverage $(55.4 \%$ vs $46.1 \% ; P=.003)$. Patients who underwent first-line ET had a longer mean follow-up than those who received first-line chemotherapy (11.5 vs 8.2 months; $P<.001$ ) but had fewer lines of therapy (mean, 1.7 vs $2.1 ; P<.001): 754$ patients $(89.4 \%)$ who received first-line ET had $<3$ lines of ET. Mean duration of firstline therapy was longer in patients who received firstline ET, but was similar in later lines (first-line, 5.7 vs 3.1 months, $P<.001$; second-line, 4.4 vs 3.5 months, $P=.347$; third-line, 3.7 vs $3.0, P=.265$; fourth-line, 2.6 vs $2.3, P=.836)$. Across all patients, the proportion using ET decreased in subsequent lines, although everolimus use increased in later lines (first-, second-, third-, and fourth-line use, respectively, with anastrozole or letrozole was $38.7 \%, 21.2 \%, 20.0 \%$, and $12.0 \%$; for everolimus was $3.0 \%, 9.3 \%, 10.5 \%$, and $13.9 \%$; for exemestane was $4.1 \%, 3.7 \%, 5.8 \%$, and $9.3 \%$; and for fulvestrant was $11.7 \%, 8.8 \%, 7.6 \%$, and $6.5 \%)$. Conclusions: Among postmenopausal women with $\mathrm{HR}^{+} /$ HER2- $\mathrm{mBC}$, approximately two-thirds of patients received first-line ET. Although nonsteroidal aromatase inhibitors (NSAIs; anastrozole or letrozole) may be routinely prescribed in early lines, a substantial proportion of patients in this study continued to use NSAIs for consecutive lines either separated by treatment gaps of $>90$ days or switched back to NSAIs in subsequent lines. Treatment duration after first-line therapy decreased considerably, suggesting limited efficacy. With 
an increasing number of therapies for $\mathrm{mBC}$ available, there is room for treatment optimization, especially after first-line ET.

\section{Outcomes and Health Services Research \\ AB2015-16. Cost and Health Outcomes of Continuation Rules for Second-Line BCR-ABL TKI Use for Patients With CML \\ John Whalen, BSa; Ipek Özer Stillman, MSa; \\ Apoorva Ambavane, MPH; Eugene Felber, PhD, MSPH ; and Bjorn Bolinder, MSc, MBA ${ }^{\mathrm{b}}$ \\ ${ }^{a}$ Evidera, Inc., and ${ }^{b}$ Bristol-Myers Squibb}

Background: NCCN Guidelines for Chronic Myelogenous Leukemia (CML) recommend tyrosine kinase inhibitor (TKI) continuation for patients with chronic-phase CML with a complete cytogenetic response (CCyR) or major molecular response (MMR) within 3 to 18 months; otherwise, patients may benefit from an alternate TKI. Existing economic evaluations do not consider early switching as a result of inadequate response. This study evaluated the cost and health outcomes of TKI sequences with various treatment continuation rules. Methods: The analysis considered patients resistant or intolerant to first-line imatinib. A cohort Markov model was developed to estimate the lifetime costs and quality-adjusted life years (QALYs) for 6 TKI sequences (Table 1). The model starts with second-line TKIs, and patients who experienced a partial cytogenetic response $(\mathrm{PCy})$ or no response are switched to the next line of therapy at 3, 6, 12, or 18 months. Patients may also switch TKI at any time in the model because of toxicity or loss of response. After thirdline TKI therapy, patients enter a "no TKI, chronic phase" health state. At any point, patients may experience disease transformation or death. Efficacy data were based on published trials, with assumptions and interpolation used to address data gaps. Drug acquisition, monitoring, toxicity and disease management costs, and utility estimates were based on FDA labels and publicly available data. Results: Switching patients with less than a PCyR at 3 months and a PCyR at 12 months resulted in the fewest QALYs and the lowest lifetime costs when compared with a 6-month switching strategy for patients with less than a PCyR and an 18-month switch for those with less than a CCyR. For patients whose disease was imatinib-resistant, a later switch increased average QALYs by 2.81 and costs by $\$ 138,071$. For patients with imatinib-intolerant disease, switching later increased average QALYs by 2.59 and costs by $\$ 148,402$. Patients treated with second-line dasatinib achieved a $\mathrm{PCyR}$ or $\mathrm{CCyR}$ more rapidly and stayed on treatment longer, resulting in higher QALYs and costs. Conclusions: This analysis indicates that survival benefit (measured by QALYs) may be reached using treatments with a more rapid response achievement, and by minimizing suboptimal responses requiring the need to switch therapies. There is a need to try alternate therapies for nonresponders, but the potential for slower response achievement in second-line treatment needs to be recognized.

\section{AB2015-16. Table 1: TKI Sequences}

\begin{tabular}{|c|c|c|c|c|c|}
\hline \multicolumn{2}{|c|}{$\begin{array}{l}\text { TKI Continuation } \\
\text { Requirement }\end{array}$} & \multicolumn{2}{|c|}{$\begin{array}{l}\geq \mathrm{PCyR} \text { at } 3 \mathrm{mo} \\
\geq \mathrm{CCyR} \text { at } 12 \mathrm{mo}\end{array}$} & \multicolumn{2}{|c|}{$\begin{array}{l}\geq \mathrm{PCyR} \text { at } 6 \mathrm{mo} \\
\geq \mathrm{CCyR} \text { at } 18 \mathrm{mo}\end{array}$} \\
\hline \multicolumn{2}{|c|}{$\begin{array}{l}\text { Patient Population } \\
\& \text { Sequence (after } \\
\text { failure of first-line } \\
\text { imatinib) }\end{array}$} & $\begin{array}{l}\text { Total Cost } \\
\text { (\$) }\end{array}$ & QALYS & $\begin{array}{l}\text { Total Cost } \\
\text { (\$) }\end{array}$ & QALYS \\
\hline \multirow{4}{*}{$\begin{array}{l}\text { Imatinib- } \\
\text { resistant }\end{array}$} & HDI-NIL & 208,536 & 2.1 & 344,283 & 4.9 \\
\hline & HDI-DAS & 207,092 & 2.1 & 343,492 & 4.9 \\
\hline & NIL-DAS & 352,283 & 3.8 & 492,560 & 6.6 \\
\hline & DAS-NIL & 392,739 & 4.2 & 539,608 & 6.9 \\
\hline \multirow{4}{*}{$\begin{array}{l}\text { Imatinib- } \\
\text { intolerant }\end{array}$} & NIL-BOS & 416,715 & 4.4 & 558,332 & 7.0 \\
\hline & DAS-BOS & 477,827 & 5.0 & 623,787 & 7.5 \\
\hline & NIL-DAS & 421,033 & 4.5 & 572,259 & 7.2 \\
\hline & DAS-NIL & 482,476 & 5.1 & 637,282 & 7.7 \\
\hline
\end{tabular}

Abbreviations: BOS, bosutinib; DAS, dasatinib; HDI, high-dose imatinib; NIL, nilotinib.

Outcomes and Health Services Research

AB2015-17. Does Hospital Proficiency Vary for Melanoma Sentinel Lymph Node Biopsies? An Evaluation of Hospital-Level Adjusted Node-Positivity Rates and Outcomes

Christine V. Kinnier, MDa, ${ }^{\mathrm{a}, \mathrm{b}}$; ; Jennifer L. Paruch, MD ${ }^{\mathrm{d}, \mathrm{e}}$; Allison R. Dahlke, MPHa,b; Jeffrey D. Wayne, MDa,b;

Al B. Benson III, MD ${ }^{\mathrm{a}, \mathrm{b}}$; David P. Winchester, MD ${ }^{\mathrm{d}, \mathrm{f}}$; and Karl Y. Bilimoria, MD, MS ${ }^{\mathrm{a}, \mathrm{b}}$

a Robert H. Lurie Comprehensive Cancer Center, ${ }^{b}$ Northwestern University, Feinberg School of Medicine, 'Massachusetts General Hospital, 'American College of Surgeons, eUniversity of Chicago Pritzker School of Medicine, and ${ }^{f}$ NorthShore University HealthSystem

This abstract is available at J Am Coll Surg 2014;219(Suppl):S99. 